CARDIOVASCULAR MEDICINE

\title{
Acute coronary syndrome and chronic infection in the Cork coronary care case-control study
}

\author{
J Sheehan, P M Kearney, S O Sullivan, C Mongan, E Kelly, I J Perry
}

Heart 2005;91:19-22. doi: 10.1136/hrt.2003.031799

See end of article for authors' affiliations

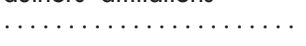

Correspondence to:

Dr Patricia M Kearney,

Department of

Epidemiology and Public

Health, University College

Cork, Cork, Ireland:

patricia.kearney@ucc.ie

Accepted 26 March 2004

.....................
Objective: To examine the association between chronic infection and cumulative burden of infection and acute coronary syndrome.

Design: The 5C (Cork coronary care case-control) study was a community based case-control study. Patients and controls underwent a standard physical examination and had blood samples taken for serological analysis for Helicobacter pylori (lgG), Chlamydia pneumoniae ( $\lg A, \lg M$, and $\lg G)$, cytomegalovirus (lgG), and herpes simplex virus types 1 and 2 (lgG).

Setting: Patients were recruited from four hospitals in Cork City and Mallow Town. Controls, individually matched on age and sex, were selected by incident density sampling from the same general practices as the referent case.

Main outcome measures: Age and sex adjusted and fully adjusted odds ratios for acute coronary syndrome by seropositivity and by increasing number of infections.

Results: Cases and controls did not differ significantly in seropositivity to $C$ pneumoniae, cytomegalovirus, herpes simplex viruses, and $H$ pylori. In unconditional logistic regression analysis adjusted for age, sex, waist to hip ratio, smoking, physical activity, alcohol consumption, and social class there was no evidence of an increasing risk for acute coronary syndrome with increasing burden of infection.

Conclusions: The findings do not support an association between specific infectious agents and acute coronary syndrome and do not provide evidence of a burden of infection effect.
C hronic infection has been proposed as a possible causative agent in the development of acute coronary syndrome. ${ }^{1}$ Many pathogens have been implicated in the development of acute coronary syndrome including Chlamydia pneumoniae, cytomegalovirus, Helicobacter pylori, and herpes simplex virus (HSV) types 1 and $2 .^{2-5}$

A recent meta-analysis of observational studies did not support the hypothesis linking specific microorganisms to acute coronary syndrome. ${ }^{6}$ Similarly, promising preliminary findings from randomised controlled trials of antimicrobial agents have not been reproduced in more recent work..$^{7-9}$ More recently attention has broadened to include the burden of infection hypothesis, the concept that it is not one specific infection per se but the cumulative burden of infection that increases a person's risk of acute coronary syndrome. ${ }^{10}{ }^{11}$

Diseases of the circulatory system, of which coronary heart disease is the largest component, account for $41 \%$ of deaths in Ireland, and Ireland has one of the highest rates of mortality for cardiovascular disease in the European Union. ${ }^{12}{ }^{13}$ Acute coronary syndrome, defined as acute myocardial infarction or unstable angina, is one of the most common reasons for emergency admission to hospital in Ireland. ${ }^{14}$ The aim of this study was to examine the association between chronic infection, specifically seropositivity for $C$ pneumoniae, cytomegalovirus, HSV types 1 and 2, and $H$ pylori, and acute coronary syndrome and to assess the odds of acute coronary syndrome with increasing burden of infection.

\section{METHODS}

Design, participants, and methods of data collection The 5C (Cork coronary care case-control) study was a community based case-control study. The study was carried out in four Cork area hospitals and 86 general practices in Cork and Kerry. The case group $(\mathrm{n}=227)$ was recruited from consecutive patients aged between 35-74 years (inclusive) admitted with a first acute myocardial infarction or first event unstable angina, defined on the basis of standard World Health Organization criteria, to four Cork areas hospitals over a 26 month period. ${ }^{15}$ Controls were matched by age (within five years) and sex and were selected by incident density sampling from the age-sex registers or practice lists of the general practices from which the cases were referred (86 practices). In the original study protocol, the study had a power of $90 \%$ to detect an odds ratio of 2 for the association between $C$ pneumoniae and acute coronary syndrome (assuming a C pneumoniae seropositivity of $50 \%$ in controls and a sample size of 300 cases and 600 controls). The power of the study was recalculated based on the achieved sample size and the power was $80 \%$ to detect an odds ratio of 1.7 for the association between $C$ pneumoniae and acute coronary syndrome.

\section{Exclusion criteria}

The following characteristics were exclusion criteria for the study: (1) age $<35$ years or $>74$ years; (2) documented history of previous myocardial infarction, angina, other heart disease or stroke; (3) previous (old) 12 lead ECG showing pathological Q waves, ST segment deviation, $\mathrm{T}$ wave inversion, bundle branch or atrioventricular block, or tachyarrhythmia other than isolated extrasystoles; (4) documented coagulation disorder or myeloproliferative

Abbreviations: 5C, Cork coronary care case-control; ACADEMIC, azithromycin in coronary artery disease: elimination of myocardial infection with chlamydia; $\mathrm{BRHS}$, British regional heart study; $\mathrm{Cl}$, confidence interval; EIU, enzyme immune units; ELISA, enzyme linked immunosorbent assay; HSV, herpes simplex virus; ISAR-3, intracoronary stenting and antithrombotic regimen; ROXIS, randomised trial of roxithromycin in non-Q-wave coronary syndromes; STAMINA, South Thames trial of antibiotics in myocardial infarction and unstable angina; WIZARD, weekly intervention with Zithromax for atherosclerosis and its related disorders 
disease; (5) severe mental or physical disability; (6) severe or life threatening intercurrent illness; (7) residence outside the Southern Health Board area; (8) unwillingness to participate in the study; and (9) no contact telephone number. The same exclusion criteria applied for patients and controls. Patients were interviewed, examined, and had blood samples taken between 3-10 days after admission. Forty nine per cent of the age and sex matched controls were examined within one month of the patients (median 33 days, range $1-417$ days).

\section{Questionnaire and physical measurements}

The questionnaire was adapted from the BRHS (British regional heart study) baseline screening questionnaire, with permission from the BRHS study group. It addressed in detail current and previous (lifelong) exposures under the following headings: occupational and economic status, patterns of physical activity, alcohol intake, smoking habits and use of medication, and family history of cardiovascular disease, hypertension, and diabetes mellitus. Smoking status was classified into three categories: never smoker, former smoker, and current smoker. Participants were divided by alcohol consumption into heavy drinkers (men $>21$ units a week and women $>14$ units a week), moderate drinkers (men who drank alcohol but drank $<21$ units a week and women who drank alcohol but drank $<14$ units a week), and nondrinkers. Social class was classified from current or former primary longest held occupation or by spouse's occupation for participants who gave no occupation or worked in the home. Social class was divided into nine occupational groupings according to standardised occupational coding lists from the Central Statistics Office of Ireland. ${ }^{16}$ Both groups were measured for height, weight, and waist to hip ratio. These were measured by the same standardised methods used in the Cork and Kerry study. ${ }^{17}$

\section{Measurement of chronic infections}

$H$ pylori antibody status was tested in the microbiology laboratory in the Mercy Hospital, Cork, with the Biomaster Assay Junior. The assay used was the Premier $H$ pylori assay kit (Meridian Bioscience Inc, Cincinnati, Ohio, USA). This assay has a sensitivity of $88 \%$ and a specificity of $96 \%{ }^{18}$ Serum was tested for cytomegalovirus infection with the Biotest anti-human cytomegalovirus recombinant IgG enzyme linked immunosorbent assay (ELISA) in the National Virus Reference Laboratory, University College Dublin. The Biotest IgG ELISA has a sensitivity of $100 \%$ and a specificity of $99 \%$.

C pneumoniae antibody status was measured in the Virus Reference Laboratory in University College Dublin with an ELISA. Antibody status to C pneumoniae was determined for
IgA, IgM, and IgG antibodies. The antibodies were measured in enzyme immune units (EIU). For IgA antibodies a value of $>12$ EIU was treated as positive, a value of 8-12 EIU was equivocal, and a value of $<8$ EIU was negative. For IgG antibodies $>45$ EIU was positive, 30-45 EIU was equivocal, and $<30$ EIU was negative. For IgM $>1.1$ EIU was positive, $0.5-1.1$ EIU was equivocal, and $<0.5$ was negative. For the purposes of the analysis equivocal results were regarded as negative. The Labsystems enzyme immunoassay has a sensitivity of $91 \%$ and $85 \%$ and specificity of $80 \%$ and $88 \%$ for IgG and IgA, respectively.

HSV types 1 and 2 were measured in the National Virus Reference Laboratory in University College Dublin with the Diamedix enzyme immunoassay (Diamedix, Miami, Florida, USA). The Diamedix enzyme immunoassay has a sensitivity of $100 \%$ and a specificity of $92.7 \% .^{19}$

\section{Statistical analysis}

The data were entered on to an OmniForm database (ScanSoft, Peabody, Massachusetts, USA) and analysed with Minitab for Windows (version 13; Minitab, State College, Pennsylvania, USA) and SAS version 10 (SAS Institute, Cary, North Carolina, USA). Adjusted means were compared by the analysis of variance-general linear model function on Minitab. Proportions were compared by $\chi^{2}$ tests. Matched and unmatched analyses were conducted by, respectively, unconditional and conditional logistic regression. Odds ratios were calculated as the estimate of association. The results of the analyses were similar; therefore, only the unmatched analyses are reported here.

Ethics

The study complies with the Declaration of Helsinki. Patients gave informed consent for the study, which has been

Table 2 Seropositivity for Chlamydia pneumoniae, Helicobacter pylori, cytomegalovirus, and herpes simplex virus types 1 and 2 in patients and controls

\begin{tabular}{llll}
\hline Antibody response & $\begin{array}{l}\text { Patients } \\
(\mathbf{n}=\mathbf{2 2 7})\end{array}$ & $\begin{array}{l}\text { Controls } \\
(\mathbf{n}=\mathbf{2 7 7})\end{array}$ & $\mathbf{p}$ Value \\
\hline C pneumoniae (lgG) & $117(52.0)$ & $136(50.6)$ & 0.20 \\
C pneumoniae (lgA) & $122(54.2)$ & $136(50.7)$ & 0.44 \\
C pneumoniae (lgM) & $8(1.8)$ & $15(1.5)$ & 0.29 \\
H pylori (lgG) & $139(62.3)$ & $162(61.4)$ & 0.80 \\
Cytomegalovirus (lgG) & $103(45.8)$ & $104(38.7)$ & 0.10 \\
Herpes simplex (lgG) & $207(92.0)$ & $243(90.3)$ & 0.50 \\
\hline & & & \\
$\mathrm{n}(\%)$. & & &
\end{tabular}

Table 1 Baseline physical characteristics of patients and controls

\begin{tabular}{|c|c|c|c|}
\hline Variable & Patients ( $n=227$ ) & Controls $(n=277)$ & p Value \\
\hline Age (years) & 59.4 (58.2 to 60.7$)$ & $59.0(57.9$ to 60.1$)$ & 0.63 \\
\hline Height $(\mathrm{cm})$ & $168.2(167.1$ to 169.4$)$ & $170.0(169.1$ to 171.0$)$ & 0.02 \\
\hline Weight (kg) & $79.6(77.7$ to 81.6$)$ & $79.0(77.5$ to 80.5$)$ & 0.60 \\
\hline BMI $\left(\mathrm{kg} / \mathrm{m}^{2}\right)$ & 28.1 (27.5 to 28.6$)$ & $27.3(26.8$ to 27.7$)$ & 0.04 \\
\hline Waist circumference $(\mathrm{cm})$ & 97.5 (95.9 to 99.2$)$ & $94.4(93.0$ to 95.8$)$ & 0.01 \\
\hline Hip circumference $(\mathrm{cm})$ & $102.3(100.9$ to 103.6$)$ & $104.6(103.7$ to 105.4$)$ & 0.01 \\
\hline Waist to hip ratio & $0.95(0.95$ to 0.95$)$ & $0.90(0.89$ to 0.91$)$ & $<0.01$ \\
\hline Men & $162(71.4 \%)$ & $208(75.1 \%)$ & 0.35 \\
\hline Current smokers & $81(36.2 \%)$ & $45(16.2 \%)$ & $<0.01$ \\
\hline Heavy drinkers & $39(18.1 \%)$ & $24(9.8 \%)$ & $<0.01$ \\
\hline Social class $1+2$ & $24(10.6 \%)$ & $46(16.6 \%)$ & 0.05 \\
\hline Self reported hypertension & $59(26.0 \%)$ & $82(29.6 \%)$ & 0.04 \\
\hline Self reported diabetes mellitus & $7(3.1 \%)$ & $5(1.8 \%)$ & 0.36 \\
\hline Family history of CVD & $123(57.5 \%)$ & $113(42.0 \%)$ & $<0.01$ \\
\hline
\end{tabular}


Table 3 Age and sex adjusted and fully adjusted odds ratios (OR) and $95 \% \mathrm{Cl}$ for acute coronary syndrome by seropositivity (lgG) to Chlamydia pneumoniae,

Helicobacter pylori, cytomegalovirus, and herpes simplex virus types 1 and 2 infections

\begin{tabular}{|c|c|c|}
\hline $\begin{array}{l}\text { Positive antibody } \\
\text { response (lgG) }\end{array}$ & $\begin{array}{l}\text { OR (age and sex } \\
\text { adjusted) }\end{array}$ & OR (fully adjusted) \\
\hline C pneumoniae & $1.0(1.0$ to 1.0$)$ & 1.0 (1.0 to 1.0$)$ \\
\hline$H$ pylori & $1.0(0.9$ to 1.1$)$ & $0.9(0.8$ to 1.0$)$ \\
\hline Cytomegalovirus & $0.7(0.5$ to 1.1$)$ & $0.7(0.5$ to 1.1$)$ \\
\hline Herpes simplex & $0.8(0.4$ to 1.5$)$ & 0.7 (0.3 to 1.5$)$ \\
\hline
\end{tabular}

Adjusted for age, sex, smoking, social class, exercise, alcohol use, and waist to hip ratio.

considered and approved by the Cork Teaching Hospitals research ethics committee.

\section{RESULTS}

The 5C study achieved a response rate of $94 \%$ for patients ( 227 of 241 potential cases) and $73 \%$ for controls ( 277 out of 377). Table 1 summarises the main baseline cardiovascular risk factors for both groups. The majority of patients were men (71\% v 29\% women). The ages of patients and controls were similar (59.4 years $v 59.0$ years, $\mathrm{p}=0.63$ ), reflecting their matching. There was a significant difference in height with patients being shorter than controls, even after adjustment for age and sex $(168.2 \mathrm{~cm} v 170.0 \mathrm{~cm}$, $\mathrm{p}=0.02)$. The weights of patients and controls were similar $(79.6 \mathrm{~kg} v 79.0 \mathrm{~kg}, \mathrm{p}=0.60)$. There was a significant difference between patients and controls with regard to average body mass index $\left(28.1 \mathrm{~kg} / \mathrm{m}^{2} \vee 27.3 \mathrm{~kg} / \mathrm{m}^{2}\right.$, $\mathrm{p}=0.04)$. There was also a highly significant difference between groups with regard to waist to hip ratio $(0.95 v 0.90$, $\mathrm{p}=0.00)$. A higher proportion of patients were smokers, were heavy drinkers, and had a family history of cardiovascular disease $(36.2 \% \vee 16.2 \%, p=0.00 ; 18.1 \% \vee 9.8 \%$, $\mathrm{p}=0.01$; and $57.5 \% \vee 42.0 \%, \mathrm{p}=0.00$, respectively).

Table 2 outlines the proportion of patients and controls who were seropositive to $H$ pylori, cytomegalovirus, HSV types 1 and 2, and $C$ pneumoniae. There were no significant differences between patients and controls with regard to seropositivity to $H$ pylori ( $60 \%$ v $61 \%$ ), cytomegalovirus ( $46 \%$ v $39 \%)$, and HSV types 1 and $2(92 \% v 90 \%)$. Both patients and controls had a similar serological response for all three classes of C pneumoniae antibody, with $54 \%$ of patients and $51 \%$ of controls being positive for IgA antibodies, $2 \%$ of patients and $1 \%$ of controls positive for $\operatorname{IgM}$ antibodies, and 52\% of patients and $51 \%$ of controls positive for IgG antibodies.

Table 3 outlines the age and sex adjusted and fully adjusted (adjusted for age, sex, social class, waist to hip ratio, smoking, alcohol, and exercise) odds ratios for acute coronary syndrome by seropositivity (IgG) to C pneumoniae,

Table 4 Age and sex adjusted and fully adjusted OR and $95 \% \mathrm{Cl}$ for acute coronary syndrome by increasing number of chronic infections

\begin{tabular}{lll}
\hline $\begin{array}{l}\text { Number of chronic } \\
\text { infections }\end{array}$ & $\begin{array}{l}\text { OR (age and } \\
\text { sex adjusted) }\end{array}$ & OR (fully adjusted) \\
\hline 0 & 1.0 & 1.0 \\
1 & $1.3(0.3$ to 6.3$)$ & $0.9(0.1$ to 6.9$)$ \\
2 & $1.2(0.3$ to 5.4$)$ & $0.7(0.1$ to 5.0$)$ \\
3 & $1.0(0.2$ to 4.6$)$ & $0.6(0.1$ to 4.5$)$ \\
4 & $1.2(0.2$ to 5.6$)$ & $0.9(0.1$ to 6.5$)$ \\
\hline
\end{tabular}

Adjusted for age, sex, smoking, social class, exercise, alcohol use, and waist to hip ratio
H pylori, cytomegalovirus, and HSV types 1 and 2 infections. There was no evidence of an increased risk of acute coronary syndrome with any of the four infections. The age and sex adjusted and fully adjusted odds ratios of acute coronary syndrome for patients who were seropositive (by IgG antibodies) to $C$ pneumoniae were both 1.0. Similar results were found for the association of acute coronary syndrome with IgA and IgM seropositivity to $C$ pneumoniae infection. For $H$ pylori the age and sex adjusted and fully adjusted odds ratios for acute coronary syndrome were 1.0 (95\% confidence interval (CI) 0.9 to 1.1 ) and 0.9 (95\% CI 0.8 to 1.0 ). The unadjusted odds ratio for acute coronary syndrome for cytomegalovirus and for HSV types 1 and 2 was 0.7 (95\% CI 0.5 to 1.1 ) and 0.8 (95\% CI 0.3 to 1.5 ), respectively. The adjusted odds ratios were similar, 0.7 (95\% CI 0.5 to 1.1) and 0.7 (95\% CI 0.3 to 1.5 ), for cytomegalovirus and HSV types 1 and 2 , respectively.

Table 4 outlines the age and sex adjusted and fully adjusted odds ratios for acute coronary syndrome by increasing number of chronic infections (as defined by seropositivity (IgG) to $H$ pylori, cytomegalovirus, HSV types 1 and 2, and $C$ pneumoniae) compared with no chronic infection. There was no evidence of an increased risk of acute coronary syndrome with increasing number of chronic infections. The test for trend was also non-significant.

\section{DISCUSSION}

In this community based case-control study we found no evidence of an association between acute coronary syndrome and $C$ pneumoniae, $H$ pylori, cytomegalovirus, and HSV types 1 and 2 infections.

The findings with regard to the individual organisms are consistent with previous studies. A meta-analysis performed by Danesh and colleagues ${ }^{6}$ in 2000 identified 15 prospective studies of $C$ pneumoniae IgG titres and coronary heart disease. The partially adjusted odds ratio for coronary heart disease for all 15 studies combined was 1.19 (95\% CI 0.99 to 1.41 ). Adjustment for adult and childhood socioeconomic status did not significantly alter the results. In a meta-analysis examining the association between cytomegalovirus infection and coronary heart disease, Danesh and colleagues ${ }^{20}$ found no association between seropositivity to cytomegalovirus infection and coronary heart disease (odds ratio 0.91, 95\% CI 0.69 to 1.19). The evidence to date on the association between $H$ pylori infection and coronary heart disease has been inconclusive due to contradictory results from different studies. $^{21-23}$ Similarly no clear association between HSV infection and coronary heart disease has emerged. ${ }^{24-26}$

Several randomised controlled trials have investigated the effects of antimicrobial agents on the risk of recurrent coronary heart disease, including ROXIS (randomised trial of roxithromycin in non-Q-wave coronary syndromes), ACADEMIC (azithromycin in coronary artery disease: elimination of myocardial infection with chlamydia), ISAR-3 (intracoronary stenting and antithrombotic regimen 3), STAMINA (South Thames trial of antibiotics in myocardial infarction and unstable angina), and WIZARD (weekly intervention with Zithromax for atherosclerosis and its related disorders)..$^{792-29}$ Overall in the trials antibiotics have not had a beneficial effect in the treatment of coronary heart disease.

Some studies have reported an association between number of infections and risk of coronary heart disease. Espinola-Klein and colleagues ${ }^{30}$ assessed the impact of infectious burden on extent and long term prognosis of atherosclerosis and reported a positive association between increasing level of seropositivity to infection and risk of atherosclerosis. Zhu and colleagues ${ }^{31}$ and Rupprecht and 
associates $^{32}$ reported similar findings, though the numbers were small.

\section{Conclusion}

In this community based case-control study we found no association between $C$ pneumoniae, $H$ pylori, cytomegalovirus, and HSV types 1 and 2 infections and acute coronary syndrome. We also found no evidence of a burden of infection effect.

\section{ACKNOWLEDGEMENTS}

The authors acknowledge the Health Research Board and the Irish Heart Foundation for providing funding for this project.

\section{Authors' affiliations}

J Sheehan, P M Kearney, S O Sullivan, I J Perry, Department of Epidemiology and Public Health, University College Cork, Cork, Ireland C Mongan, E Kelly, National Virus Reference Laboratory, University College Dublin, Dublin, Ireland

Conflicts of interest: None

\section{REFERENCES}

1 Danesh J, Collins R, Peto R. Chronic infections and coronary heart disease: is there a link? Lancet 1997:350:430-6.

2 Saikku $\mathbf{P}$, Leinonen $M$, Matilla $K$, et al. Serological evidence of an association of a novel chlamydia, TWAR, with chronic coronary heart disease and acute myocardial infarction. Lancet 1988;ii:983-6.

3 Adam E, Melnick JL, Probtsfield JL, et al. High levels of cytomegalovirus antibody in patients requiring vascular surgery for atherosclerosis. Lancet 1987;ii:291-3.

4 Danesh J, Muir J, Wong YK, et al. Risk factors for coronary heart disease and acute-phase proteins: a population-based study. Eur Heart J 1999;20:954-9.

5 Siscovick DS, Schwartz SM, Corey L, et al. Chlamydia pneumoniae, herpes simplex virus type 1, and cytomegalovirus and incident myocardial infarction and coronary heart disease death in older adults: the cardiovascular health study. Circulation 2000;102:2335-40.

6 Danesh J, Whincup P, Walker $M$, et al. Chlamydia pneumoniae lgG titres and coronary heart disease: prospective study and meta-analysis. $B M$ 2000;321:208-13.

7 Gurfinkel E, Bozovich G, Daroca A, et al. Randomised trial of roxithromycin in non-Q-wave coronary syndromes: ROXIS pilot study. ROXIS study group. Lancet 1997;350:404-7.

8 Ferguson JJ. Meeting highlights: highlights of the 51 st annual scientific sessions of the American College of Cardiology. Atlanta, Georgia, USA. March 17-20, 2002. Circulation 2002;106:E24-30.

9 O'Connor CM, Dunne MW, Pfeffer MA. Azithromycin for the secondary prevention of coronary heart disease events. The WIZARD study: a randomized controlled trial, JAMA 2003:290:1459-66.

10 O'Donnell CJ, Levy D. Weighing the evidence for infection as a risk factor for coronary heart disease. Curr Cardiol Rep 2000;2:280-7

11 Danesh J, Whincup P, Walker $M$, et al. Low grade inflammation and coronary heart disease: prospective study and updated meta-analyses. BMJ 2000;321:199-204.
12 Anon. Health strategy: quality and fairness. Dublin: Department of Health, 2001:1-700.

13 Anon. Health for all database. Geneva: World Health Organization, 2001

14 Anon. Activity in acute public hospitals in Ireland 1990-1999. Dublin: HIPE and NPRS Unit, The Economic and Social Research Institute, 2002:188-98.

15 Anon. World Health Organization Expert Committee. Hypertension and coronary heart disease: classification and criteria for epidemiological studies. Geneva: World Health Organization, 1959.

16 Anon. Census 96: preliminary data. Dublin: Central Statistics Office, 1997.

17 Creagh D, Neilson S, Collins A, et al. Established cardiovascular diseases and CVD risk factors in a primary care population of middle-aged Irish men and women. Ir Med J 2002;95:298-301.

18 Talley NJ, Kost L, Haddad A, et al. Comparison of commercial serological tests for detection of Helicobacter pylori antibodies. J Clin Microbiol 1992;30:3146-50.

19 Martins TB, Woolstenhulme RD, Jaskowski TD, et al. Comparison of four enzyme immunoassays with a western blot assay for the determination of type-specific antibodies to herpes simplex virus. Am J Clin Pathol 2001;115:272-7

20 Danesh J. Coronary heart disease, Helicobacter pylori, dental disease, Chlamydia pneumoniae, and cytomegalovirus: meta-analyses of prospective studies. Am Heart J 1999:138:S434-7.

21 Lip GH, Wise R, Beevers G. Association of Helicobacter pylori infection with coronary heart disease: study shows association between $\mathrm{H}$ pylori infection and hypertension. BMJ 1996;312:250-1.

22 Brenner $\mathbf{H}$, Berg $G$, Frohlich $M$, et al. Chronic infection with Helicobacter pylori does not provoke major systemic inflammation in healthy adults: results from a large population-based study. Atherosclerosis 1999;147:399-403.

23 Whincup P, Danesh J, Walker M, et al. Prospective study of potentially virulent strains of Helicobacter pylori and coronary heart disease in middle-aged men. Circulation 2000;101:1647-52.

24 Sorlie PD, Adam E, Melnick SL, et al. Cytomegalovirus/herpesvirus and carotid atherosclerosis: the ARIC study. J Med Virol 1994;42:33-7.

25 Ridker PM, Hennekens CH, Stampfers MJ, et al. Prospective study of herpes simplex virus, cytomegalovirus, and the risk of future myocardial infarction and stroke. Circulation 1998;98:2796-9.

26 Roivainen M, Viik-Kajander M, Palosuo T, et al. Infections, inflammation, and the risk of coronary heart disease. Circulation 2000;101:252-7.

27 Anderson JL, Muhlestein JB, Carlquist J, et al. Randomized secondary prevention trial of azithromycin in patients with coronary artery disease and serological evidence for Chlamydia pneumoniae infection: the azithromycin in coronary artery disease: elimination of myocardial infection with chlamydia (ACADEMIC) study. Circulation 1999:99:1540-7.

28 Neumann F, Kastrati A, Miethke T, et al. Treatment of Chlamydia pneumoniae infection with roxithromycin and effect on neointima proliferation after coronary stent placement (ISAR-3): a randomised, double-blind, placebocontrolled trial. Lancet 2001:357:2085-9.

29 Stone AF, Mendall MA, Kaski JC, et al. Effect of treatment for Chlamydia pneumoniae and Helicobacter pylori on markers of inflammation and cardiac events in patients with acute coronary syndromes: South Thames trial of antibiotics in myocardial infarction and unstable angina (STAMINA). Circulation 2002; 106:1219-23.

30 Espinola-Klein C, Rupprecht HJ, Blankenberg S, et al. Impact of infectious burden on extent and long-term prognosis of atherosclerosis. Circulation 2002;105:15-21.

31 Zhu J, Nieto FJ, Horne BD, et al. Prospective study of pathogen burden and risk of myocardial infarction or death. Circulation 2001;103:45-51.

32 Rupprecht HJ, Blankenberg S, Bickel C, et al. Impact of viral and bacterial infectious burden on long-term prognosis in patients with coronary artery disease. Circulation 2001;104:25-31.

\section{ELECTRONIC PAGES}

\section{Heart Online case reports: www.heartjnl.com}

$\mathrm{T}$ he following electronic only article is published in conjunction with this issue of Heart.

\section{Intracerebral haematoma masquerading as ventricular standstill}

\section{A K J Mandal, S Baltsezak, C G Missouris}

An 82 year old man was referred to the emergency room by his general practitioner for a right frontoparietal headache. The preceding day he had tripped and fallen, hitting the back of his head on the floor. Computed tomography showed a cortical contre coup haematoma. In view of ventricular standstill noted on ECG, a temporary pacing wire was inserted and a dual chamber permanent pacemaker was subsequently implanted. Intracerebral bleeding was treated conservatively and the patient made a good recovery. All patients admitted with head injury and sinus bradycardia or sinus arrest should be nursed at $15^{\circ}$ to $30^{\circ}$ with instructions to avoid the head up and supine positions. Furthermore, brain CT should be promptly recorded to assess for intracerebral haematoma and raised intracranial pressure and, if they are confirmed, these patients with cardiovascular compromise should benefit from close collaboration between neurosurgeon and cardiologist. Urgent pacing should be considered for all patients with head injury who experience symptomatic bradycardia or ventricular standstill.

(Heart 2005;91:el) www.heartjnl.com/cgi/content/full/91/1/el 